\title{
Collaboration of perioperative management in an adult patient with 22 q 11.2 deletion syndrome: A case report
}

Mikiko Ito ${ }^{1}$, Tatsuya Tokura ${ }^{2}$, Tomoya Miyauchi ${ }^{3}$, Aiji Sato (Boku) ${ }^{4}$, Hiroyuki Kimura ${ }^{2}$, Hayami Tsuchihashi ${ }^{5}$, and Yoshiko Katayama ${ }^{6}$

${ }^{1}$ School of Dentistry, Aichi-Gakuin University

${ }^{2}$ Nagoya University Graduate School of Medicine

${ }^{3}$ Kachi Memorial Hospital

${ }^{4}$ Aichi Gakuin University School of Dentistry

${ }^{5}$ School of Dentistry, Aichi-Gakuin University

${ }^{6}$ School of Dentistry, Aichi-Gakuin University

October 19, 2021

\begin{abstract}
22 q 11.2 deletion syndrome is a genetic disease associated with a high rate of various psychiatric disorders in addition to multiple physical diseases. This case report suggested that a multidisciplinary team approach is important for perioperative management of patient with 22 q 11.2 deletion syndrome.
\end{abstract}

\section{Title:}

Collaboration of perioperative management in an adult patient with 22 q 11.2 deletion syndrome: A case report

\section{Authors:}

Mikiko Ito D.D.S., Ph.D. ${ }^{1}$, Tatsuya Tokura M.D., Ph.D. ${ }^{2}$, Tomoya Miyauchi M.D., Ph.D. ${ }^{3}$, Aiji Sato (Boku) D.D.S., Ph.D. ${ }^{4}$, Hiroyuki Kimura M.D., Ph.D. ${ }^{2}$, Hayami Tsuchihashi D.D.S. ${ }^{1}$, Yoshiko Katayama D.D.S. ${ }^{1}$

Institutional affiliations and address for each author:

1 Department of Oral and Maxillofacial Surgery, School of Dentistry, Aichi-Gakuin

University, 2-11 Suemori-dori, Chikusa, Nagoya, Aichi 464-8651, Japan

2 Department of Psychiatry, Nagoya University Graduate School of Medicine, 65 Tsurumai, Showa, Nagoya, Aichi 466-8550, Japan

3 Department of Psychiatry, Kachi Memorial Hospital, 456 Fujimi, Minamiooshimizu, Toyohashi, Aichi 441-8132, Japan

4 Department of Anesthesiology, School of Dentistry, Aichi-Gakuin University, 2-11 Suemori-dori, Chikusa, Nagoya, Aichi 464-8651, Japan

\section{Corresponding author:}

Tatsuya Tokura M.D., Ph.D. 
Department of Psychiatry, Nagoya University Graduate School of Medicine, 65 Tsurumai, Showa, Nagoya, Aichi 466-8550, Japan

Tel: +81 52 7442282; Fax: +81 52 7442293; E-mail: tatsuyatokura@gmail.com

\title{
Key clinical message:
}

A multidisciplinary team approach is important for perioperative management of adult patient with $22 \mathrm{q}$ 11.2 deletion syndrome.

\begin{abstract}
:
22 q 11.2 deletion syndrome is a genetic disease associated with a high rate of various psychiatric disorders in addition to multiple physical diseases. This case report suggested that a multidisciplinary team approach is important for perioperative management of patient with 22 q 11.2 deletion syndrome.
\end{abstract}

\section{Keywords:}

22 q 11.2 deletion syndrome, multidisciplinary team approach, perioperative management.

\section{Introduction}

22 q 11.2 deletion syndrome (22 q 11.2 DS) is a genetic disease that is often complicated by psychiatric disorders such as intellectual disability, autism spectrum disorder, attention-deficit/hyperactivity disorder, and schizophrenia in addition to physical diseases such as congenital heart disease, palate hypoplasia, and immunodeficiency. This syndrome is said to be the most common of the chromosomal microdeletion syndromes, and a recent Danish cohort study reported that 1 in 3672 people developed it ${ }^{1}$. Although the prognosis has been said to be poor, the adult population with 22 q 11.2 DS is increasing due to advances in pediatric care ${ }^{2}$.

Acceptable standard care for individuals with 22 q 11.2 DS is a careful coordination and multidisciplinary team approach that provides access to medical services throughout their life cycle ${ }^{3}$. In this report, we successfully collaborated in the perioperative management of oral surgery in an adult $22 \mathrm{q} 11.2$ DS patient with multiple physical diseases and schizophrenia.

\section{Case Report}

The patient was a 24-year-old woman. Anal atresia and cleft palate were found at birth, and the patient took a genetic test and was diagnosed with 22 q 11.2 DS. At one month after birth, the patient was diagnosed as having aortic stenosis due to bicuspid aortic valve in the pediatric cardiology department. The patient was placed under observation, and general exercise restrictions were not necessary, but sports club activities were prohibited. The patient underwent palatoplasty at the age of 1 year and 6 months, and orthodontic treatment from the age of 9 to 20 years with a diagnosis of dental stenosis. At the age of 20, the patient got married and gave birth.

At the age of 22 , the patient was diagnosed with schizophrenia due to the patient's hallucination and delusion and was admitted to a psychiatric hospital for 10 months. The symptoms have improved by taking antipsychotic drug and modified electroconvulsive therapy. After being discharged from the hospital, the patient noticed toothache and was referred to our hospital by another dental clinic.

The patient was $148 \mathrm{~cm}$ tall, weighed $41 \mathrm{~kg}$, and had a blood pressure of 110/70 $\mathrm{mmHg}$ at the time of medical examination. There were no other problems with blood tests or electrocardiograms.

Extraoral findings of the patient showed microtia, micromandibula, micronostrils, micrognathia and trismus. The intraoral findings showed that 15, 17, 28, 34, 42, 43, 45, 46, 48 (Fédération Dentaire Internationale: FDI) were in a residual root state. The patient's oral hygiene status was extremely poor. Panoramic radiographs showed the apices of 11, 45, 46 (FDI) with round radiolucent images suggesting apical lesions (Figure). We planned to extract 10 teeth, specifically $(11,15,17,28,34,42,43,45,46,48$ (FDI) ). The risk of the procedure itself was low, but the risks arising from complications of 22 q 11.2 DS were a concern. 
The severity of aortic stenosis was unknown because the patient interrupted the periodic medical examination by self-judgement at the age of 20 . We contacted the pediatric cardiologist and obtained the general anesthesia records of this patient's maternal delivery by cesarean section three years ago.

Echocardiography at the department of cardiology of our hospital showed a pressure gradient between the left ventricle and the aorta of $20 \mathrm{mmHg}$. The severity of aortic stenosis was judged to be mild. Although there were no problems with cardiac function, the patient was at risk for infective endocarditis, and $2 \mathrm{~g}$ of ampicillin was prepared.

The dental anesthesiologist diagnosed the patient as having difficult intubation due to micrognathia and severe trismus and judged that conscious fiberscope-guided intubation under mild sedation was also impossible because the patient's psychiatric symptoms were unstable. After induction of general anesthesia, the dental anesthesiologist administered $30 \mathrm{mg}$ of rocuronium, a muscle relaxant, and performed nasotracheal intubation under fiber scope guidance. We explained the risks of anesthesia to the patient and the patient's family in advance and obtained their consent in writing.

The following risks information were provided by the attending psychiatrist: 1) discontinuation of psychotropic drugs may aggravate schizophrenia; 2) examination by an oral surgeon may increase anxiety and irritation; and 3) interactions between antipsychotics and epinephrine contained in the local anesthetics may occur. Therefore, the administration of psychotropic drugs was continued even on the night before the operation, the preoperative examination by the oral surgeon was shortened, and the local anesthetic for surgery was prepared as ferripressin-containing propitocaine hydrochloride without epinephrine.

The above risks were shared by a multidisciplinary team including oral surgeons, dental anesthesiologists, psychiatrists, cardiologists, nurses and social workers. We extracted 10 teeth as scheduled. The day after the operation, we transferred the patient to the psychiatric hospital where the patient had been hospitalized. One week later, the patient had good progress and we completed the collaboration of perioperative management.

\section{Discussion}

The course of this patient provides the following important clinical suggestions. Since the symptoms and severity of 22 q 11.2 DS vary widely among individuals, multidisciplinary medical collaboration is necessary for perioperative management.

Frequent comorbidities of 22 q 11.2 DS are multidisciplinary, including cardiac anomalies (49-83\%), palatal anomalies (69-100\%), dental skeletal anomalies (40-50\%), hypocalcemia (17-60\%), schizophrenia (6-30\%) and attention-deficit/hyperactivity disorder $(25 \%)^{4}$.

Life-threatening complications include congenital heart disease and the most common one is tetralogy of Fallot $(20-45 \%)^{5}$. Congenital heart disease adds to the complexity associated with acquired cardiovascular problems and multiorgan comorbidities, and requires perioperative management that considers immune dysfunction, thrombocytopenia and hypocalcemia ${ }^{5}$. This patient had no tetralogy of Fallot but aortic stenosis. Aortic stenosis is a rare complication of $22 \mathrm{q} 11.2 \mathrm{DS}^{5}$, but as it is a risk group for infective endocarditis. Therefore, antibiotic ampicillin was administered prophylactically. Because the severity of aortic stenosis was unknown, the risk for general anesthesia was also unknown. Through the medical cooperation system, general anesthesia records of the recent cesarean section and modified electroconvulsive therapy were received from the pediatric cardiologist and the psychiatrist.

In generally, physicians have trouble dealing with patients with psychiatric disorders, while psychiatrists have trouble dealing with patients with physical illness. Perioperative management of oral surgery for patients with schizophrenia requires a history of psychiatric treatment, assessment of psychotic symptoms and consideration of the duration of psychotropic drug withdrawal to control schizophrenic symptoms ${ }^{6,7}$. This case was also coordinated to be transferred to the psychiatric hospital where the psychiatrist worked the day after the operation in accordance with the risk information obtained from the psychiatrist.

In 22 q 11.2 DS, a high rate of palatal hypoplasia is observed, and it is necessary to devise measures for 
difficult intubation. We decided that it would be difficult to intubate this patient due to micrognathia and trismus. The patient had experienced general anesthesia in the past. At that time oral intubation by laryngeal mask was performed, because the operative field was not the oral cavity. We finally chose nasofiberscopeguided intubation after induction of general anesthesia. In patients with 22 q 11.2 DS, general anesthesia or intravenous sedation has been used for dental treatment ${ }^{8}$. However, there have been no previous reports of fiberscope-guided nasal intubation after induction of general anesthesia. Our team responded flexibly to risks with our own ideas, using precedents as a guide.

We experienced an adult patient with 22 q 11.2 DS in whom a multidisciplinary team approach was effective in collaboration of perioperative management. In the future, it is expected that the number of adult patients with 22 q 11.2 DS who require oral surgery under general anesthesia with multiple risks will increase as in this case. Since the symptoms and severity of 22 q 11.2 DS vary widely among individuals, it is suggested that accumulation of individual perioperative management reports may be clinically useful in the future.

\section{Conclusion}

We experienced an adult patient with 22 q 11.2 DS who needed to extract teeth. Through our experience with this case, we conclued that a multidisciplinary team approach is important for perioperative management of adult patient with 22 q 11.2 DS.

\section{Acknowlegements:}

This case report was supported by a governmental grant in aid for young scientists (No. 19K17108) from the Japan Society for the Promotion of Science.

\section{Conflict of interest:}

There were no conflicts of interest related to this publication.

\section{Author contributions :}

All authors contributed to the case. Preoperative management was performed by YK, MI and HT. Anesthetic planning and management were performed by AS. The first draft of the manuscript was written by MI, TM and HK. And all authors commented on previous versions of the manuscript. TT supervised the manuscript. All authors read and approved the final manscript.

\section{Consent for publication:}

Written informed consent was obtained from the patient and the patient's family for this case report.

\section{ORCID:}

Mikiko Ito: 0000-0002-4618-3688

Tatsuya Tokura (corresponding author): 0000-0002-2293-2349

\section{References:}

1. Olsen L, Sparso T, Weinsheimer SM, et al. Prevalence of rearrangements in the 22q11.2 region and population-based risk of neuropsychiatric and developmental disorders in a Danish population: a casecohort study. Lancet Psychiatry 2018;5:573-80.

2. Fung WL, Butcher NJ, Costain G, et al. Practical guidelines for managing adults with 22q11.2 deletion syndrome. Genet Med 2015;17 :599-609.

3. Habel A, Herriot R, Kumararatne D, et al. Towards a safety net for management of 22q11.2 deletion syndrome: guidelines for our times. Eur J Pediatr. 2014;173 :757-65.

4. Kobrynski LJ, Sullivan KE. Velocardiofacial syndrome, DiGeorge syndrome: the chromosome 22q11.2 deletion syndromes. Lancet 2007;370 :1443-52. 
5. Unolt M, Versacci P, Anaclerio S, et al. Congenital heart diseases and cardiovascular abnormalities in 22q11.2 deletion syndrome: From well-established knowledge to new frontiers. Am J Med Genet A $2018 ; 176: 2087-98$.

6. Karayiorgou M, Simon TJ, Gogos JA. 22q11.2 microdeletions: linking DNA structural variation to brain dysfunction and schizophrenia. Nat Rev Neurosci 2010;11 :402-16.

7. Constance LSL, Lansing MG, Khor FK, et al. Schizophrenia and anaesthesia. BMJ Case Rep 2017;23 :bcr2017221659.

8. AlQarni MA, Alharbi A, Merdad L. Dental management of a patient with 22q11.2 deletion syndrome (22q11.2DS). BMJ Case Rep 2018;18 :bcr2018225765.

\section{Figure Legends:}

Figure. Panoramic X-ray photograph at the first visit

Panoramic radiographs showed the apices of 11, 45, 46 (Federation Dentaire Internationale) with round radiolucent images suggesting apical lesions (Arrows to the lesions).

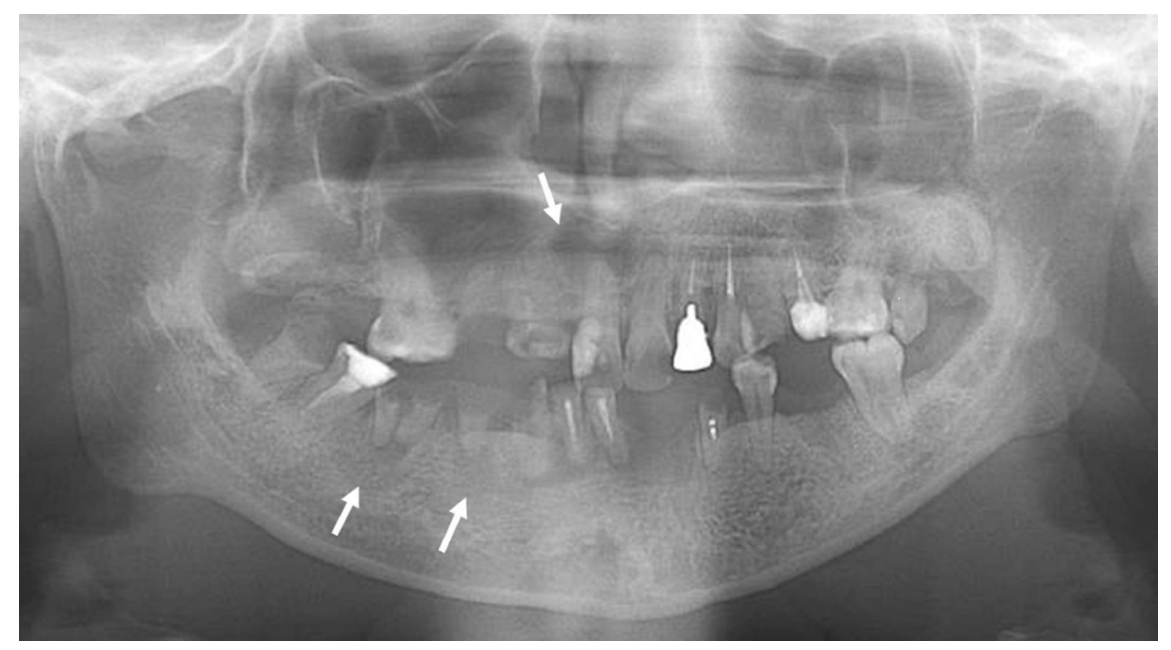

